\title{
Nível de Conhecimento dos Manipuladores de Alimentos Sobre Boas Práticas em Restaurantes Tipo Self-Service do Município de Patos - PB
}

\section{Level of Knowledge of Food Handlers on Good Practices in Self-Service Restaurants in the Municipality of Patos - PB}

\author{
Saula Virginia Alves de Lima Medeiros Confessor ${ }^{\mathrm{a}}$; Vitória Viviane Ferreira de Aquino ${ }^{\mathrm{a}}$; Aline Antas Cordeiro

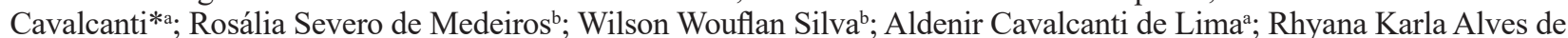 \\ Lima Medeiros ${ }^{c}$; Giuliana Amélia Freire Pereira Duarte ${ }^{b}$ Hosaneide Gomes de Araújo ${ }^{a}$; Ana Célia Rodrigues Athayde ${ }^{b}$ \\ âniversidade Federal de Campina Grande, Programa de Pós-Graduação em Stricto Sensu Ciência e Saúde Animal. PB, Brasil.

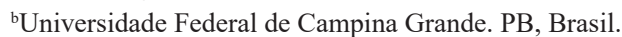 \\ 'Universidade Federal da Paraíba. PB, Brasil. \\ *E-mail: aline.antas@hotmail.com
}

\begin{abstract}
Resumo
As doenças transmitidas por alimentos tem o manipulador de alimentos como um fator de risco para sua ocorrência, quando falhas são cometidas no processo de produção dos alimentos. O presente estudo teve como objetivo determinar o nível de conhecimento dos manipuladores de alimentos dos restaurantes tipo self-service do município de Patos - PB, quanto as boas práticas de manipulação. A pesquisa se deu por meio de aplicação de um questionário estruturado contendo 34 questões relacionadas às características sociodemográficas e ao conhecimento sobre boas práticas de manipulação para 65 manipuladores de alimentos de 25 restaurantes self-service, selecionados a partir dos estabelecimentos cadastrados na Vigilância Sanitária Municipal. Na classificação do nível de conhecimento foi adotado como satisfatória uma porcentagem igual ou superior a $60 \%$ de acertos. Os dados foram analisados através da estatística descritiva. Para os dados sociodemográficos, o estudo mostrou a predominância dos seguintes fatores: sexo feminino (95,0\%), faixa etária de 40 a 49 anos (36,9\%), ensino fundamental incompleto (36,95\%), não realizam exames admissionais e/ou de rotina $(75,4 \%)$ e parasitológico de fezes $(40,0 \%)$, nunca participou de treinamento $(60,0 \%)$. O nível de conhecimento dos manipuladores foi considerado como satisfatório sendo com média de acertos de 89,16\%. Diante dos resultados obtidos, apesar dos manipuladores apresentarem um nível de conhecimento satisfatório, não o põe em prática, sendo necessária uma capacitação periódica para melhorar a qualificação profissional dos manipuladores, visando o fornecimento de alimentos seguros e minimizando os riscos de ocorrências de doenças transmitidas por alimentares.
\end{abstract}

Palavras-chave: Doenças Transmitidas por Alimentos. Manipuladores. Boas Práticas. Segurança dos Alimentos.

\begin{abstract}
The foodborne diseases have the food handler as a risk factor for its occurrence, when failures are made in the food production process. This study aimed to determine the level of knowledge of food handlers in self-service restaurants in the municipality of Patos - PB, regarding the good handling practices. The research took place through the application of a structured questionnaire containing 34 questions related to sociodemographic characteristics and knowledge about good handling practices, for 65 food handlers from 25 self-service restaurants, selected from establishments registered with the Municipal Health Surveillance. In the classification of the level of knowledge, a percentage equal to or greater than $60 \%$ of correct answers was adopted as satisfactory. The data were analyzed using descriptive statistics. For sociodemographic data, the study showed the predominance of the following factors: female gender (95,0\%), age group 40-49 years (36,9\%), incomplete elementary school (36,95\%), do not perform admission exams and/or routine $(75,4 \%)$ and parasitological stools $(40,0 \%)$, never participated in training $(60,0 \%)$. The level of knowledge of the manipulators was considered satisfactory, with an average of $89,16 \%$ correct answers. In view of the results obtained, despite the manipulators having a satisfactory level of knowledge, it does not put it into practice, requiring periodic training to improve the professional qualification of the manipulators, aiming at providing safe food and minimizing the risks of foodborne diseases.
\end{abstract}

Keywords: Foodborne Diseases. Manipulators. Good Practices. Food Safety.

\section{Introdução}

As Doenças Transmitidas por Alimentos (DTA) têm estimulado a realização de pesquisas para se identificar e analisar os agentes etiológicos e os fatores relacionados às suas causas, sendo importante para estabelecer os meios de prevenção e controle (MEDEIROS; CARVALHO; MAIA, 2017).

As DTA são causadas em sua maioria por bactérias e suas toxinas, vírus e parasitas, provenientes da ingestão de água e alimentos contaminados, sendo fatores de risco relevantes para sua ocorrência os procedimentos de manipulação, conservação de alimentos e a própria higiene pessoal dos manipuladores (BRASIL, 2017).

O manipulador é um elemento importante quando se trata de segurança dos alimentos, pois tem contato com estes, da origem até a comercialização, podendo se tornar um veículo de agentes patogênicos de doenças alimentares, quando ocorrem falhas e erros no processo de manipulação (MEDEIROS; CARVALHO; MAIA, 2017).

As capacitações dos manipuladores são importantes para a prevenção da maioria das DTA, devendo ser inclusas e contínuas, pois formam e sensibilizam os manipuladores 
sobre as técnicas adequadas na manipulação de alimentos, correção nas falhas de higiene pessoal, avaliação da saúde dos funcionários e a implementação das Boas Práticas de Manipulação (BPM), visando à qualidade e a segurança dos alimentos para os consumidores (BRASIL, 2004; CAVALLI; SALAY, 2004; CAMPOS et al., 2009; DEVIDES; MAFFEI; CATANOZI, 2014).

Os programas de treinamento devem ser teórico-práticos, ou seja, ensinar habilidades, promover a aprendizagem ativa, aumentar a percepção do risco de contaminação, considerando sempre o ambiente de trabalho, para que haja uma correlação positiva com as atitudes, práticas relatadas e práticas observadas dos manipuladores de alimentos (NIETOMONTENEGRO; BROWN; LABORDE, 2008).

$\mathrm{O}$ presente estudo teve como objetivo determinar o nível de conhecimento dos manipuladores de alimentos dos restaurantes tipo self-service do município de Patos - PB, quanto as BPM.

\section{Material e Métodos}

Para avaliar o nível de conhecimento dos manipuladores de alimentos foi realizado um estudo transversal no período de março a abril de 2019. A coleta de dados foi feita por meio da aplicação de um questionário pelo pesquisador com os manipuladores de alimentos de 25 restaurantes tipo self-service localizados em bairros diferentes no município de Patos-PB, selecionados a partir dos estabelecimentos cadastrados na Vigilância Sanitária do Município de Patos $\mathrm{PB}$, tendo participado da pesquisa todos os restaurantes tipo self-service que atendiam a este critério. Estes restaurantes eram de pequeno e médio porte, com o número de refeições por dia variando de 50 a 150, e uma média de 3 manipuladores por restaurante. O tempo médio de aplicação dos questionários foi de 15 minutos.

A amostragem foi realizada por conveniência, onde as pessoas entrevistadas foram exclusivamente os manipuladores de alimentos, tendo participado da pesquisa todos os manipuladores abordados, totalizando 65 pessoas. Os dados foram analisados através de estatística descritiva.

O questionário elaborado foi adaptado de Souza, Azevedo e Seabra (2018), e constou de 09 questões relacionadas as características sociodemográficas e 25 aos conhecimentos sobre contaminação de alimentos, DTA e BPM.

Informações foram coletadas para a classificação sociodemográfica dos manipuladores de alimentos, como: idade, sexo, escolaridade, ocupação, exames admissionais e de rotina, experiência profissional e participação em treinamentos sobre práticas adequadas de manipulação de alimentos.

Para avaliar o conhecimento sobre segurança alimentar, foram apresentadas questões de acordo com os assuntos exigidos pela legislação vigente para o treinamento de manipuladores de alimentos (BRASIL, 2004): doenças transmitidas por alimentos, higienização e higiene pessoal. $\mathrm{O}$ questionário apresentou três opções de respostas: sim, não e não sei. No caso da resposta ser não sei era considera como errada a questão.

Para classificar o nível de conhecimento foi adotado como satisfatório valores percentuais iguais ou superiores a $60 \%$ de acertos abaixo como insatisfatória (VO et al., 2015).

$\mathrm{Na}$ aplicação dos questionários foi apresentado o Termo de Consentimento Livre e Esclarecido de acordo com a Resolução No 466/2012 (BRASIL, 2012), por meio do qual foram informados sobre os riscos e benefícios do estudo e assinado pelos participantes da pesquisa.

A pesquisa foi submetida e aprovada pelo Comitê de Ética do Hospital Universitário Alcides Carneiro da Universidade Federal de Campina Grande / HUAC - UFCG - CAAE: 05100818.6.0000.5182.

\section{Resultados e Discussão}

Os manipuladores de alimentos que participaram da pesquisa $(n=65)$ eram em sua maioria do sexo feminino $(95,4 \%)$, com predomínio da faixa etária de 40 a 49 anos $(36,9 \%)$ e grau de instrução em sua maioria com o ensino fundamental incompleto (36,95\%) (Tabela 1).

A predominância do sexo feminino também foi observada por Devides, Maffei e Catanozi (2014) no estudo com manipuladores de alimentos de Araraquara - SP (63,0\%). E difere dos constatados nas pesquisas de Souza, Azevedo e Seabra (2018) em restaurantes populares do estado do Rio Grande do Norte e Mello et al. (2010) no Rio de Janeiro, onde foram observados o predomínio do sexo masculino como trabalhadores de restaurantes populares em 74,3\% e $67,0 \%$ respectivamente. Vale lembrar, que a predominância de mulheres, neste ambiente, retrata ocupações menos valorizadas e com características de emprego doméstico.

Quadro 1 - Características socioeconômicas dos manipuladores de alimentos dos restaurantes tipo self-service do município de Patos - PB, 2019

\begin{tabular}{|l|c|}
\hline \multicolumn{1}{|c|}{ Características socioeconômicas } & Frequência (\%) \\
Sexo: & 95,4 \\
\hline Feminino & 4,6 \\
\hline Masculino & 10,8 \\
\hline Idade: & 30,8 \\
\hline $18-29$ anos & 36,9 \\
\hline $30-39$ anos & 21,5 \\
\hline 40-49 anos & \\
\hline acima de 50 anos & 36,9 \\
\hline Escolaridade: & 16,9 \\
\hline ensino fundamental incompleto & 7,7 \\
\hline ensino fundamental completo & 33,8 \\
\hline ensino médio incompleto & - \\
\hline ensino médio completo & 1,6 \\
\hline ensino superior incompleto & 3,1 \\
\hline ensino superior completo & Continua... \\
\hline sem escolaridade &
\end{tabular}




\begin{tabular}{|c|c|}
\hline & Continuação... \\
\hline Características socioeconômicas & Frequência $(\%)$ \\
\hline \multicolumn{2}{|l|}{ Função: } \\
\hline Cozinheira & 40,0 \\
\hline Saladeira & 12,3 \\
\hline Auxiliar de cozinha & 47,7 \\
\hline \multicolumn{2}{|l|}{ Está com os exames admissionais em dia? } \\
\hline Sim & 24,6 \\
\hline Não & 75,4 \\
\hline \multicolumn{2}{|l|}{ Quando fez seu último exame de fezes? } \\
\hline$<6$ meses & 27,7 \\
\hline$>6$ meses & 6,2 \\
\hline 1 ano + & 26,1 \\
\hline Não realizou & 40,0 \\
\hline \multicolumn{2}{|l|}{ Se fez, lembra do resultado? } \\
\hline Sim & 100 \\
\hline Não & - \\
\hline Negativo & 100 \\
\hline \multicolumn{2}{|l|}{ Positivo } \\
\hline \multicolumn{2}{|l|}{ Quanto tempo trabalha em restaurante? } \\
\hline $0-3$ anos & 18,5 \\
\hline 4-6 anos & 27,7 \\
\hline $7-10$ anos & 13,9 \\
\hline $11-15$ anos & 21,5 \\
\hline 16-20 anos & 4,6 \\
\hline $21-35$ anos & 13,8 \\
\hline \multicolumn{2}{|c|}{ Quando foi o último treinamento de que você participou? } \\
\hline$<6$ meses & 4,6 \\
\hline 6 meses a 1 ano & 9,2 \\
\hline$>1$ ano & 26,2 \\
\hline Nunca participou & 60,0 \\
\hline
\end{tabular}

Fonte: dados da pesquisa.

A maioria dos manipuladores estava na faixa etária superior a 40 anos (58.4\%), variando de 18 a 57 anos. Observou-se que a faixa etária, pode ser um fator determinante para o exercício da profissão de manipulador de alimentos, em virtude do tempo que desempenham esta atividade.

Quanto ao nível de escolaridade, observou-se que a maioria $61,5 \%$ dos entrevistados apresentou nível de escolaridade inferior ao ensino médio. Isso porque os serviços de alimentação empregam trabalhadores sem formação profissional específica, não sendo exigida preparação mínima do manipulador. Souza, Azevedo e Seabra (2018) relataram em seu estudo que $55,7 \%$ dos manipuladores possuíam ensino médio completo e acrescenta que o nível de educação é um fator relevante quanto à segurança dos alimentos, pois esses trabalhadores precisam entender os procedimentos operacionais padronizados (POPs), e registrar suas atividades. Para Freitas, Calazans e Alchiere (2014), o nível de escolaridade é um indicador considerável da qualidade do trabalho, crescimento profissional e qualidade de vida.

Com relação às características profissionais, a maioria dos participantes trabalhava como auxiliar de cozinha (47,7\%), com tempo de trabalho na atividade entre 4 e 6 anos $(27,7 \%)$, $75,4 \%$ não realizaram exames admissionais e/ou de rotina e $40,0 \%$ não tinham feito parasitológico de fezes. Todos que realizaram os exames relataram não apresentar alterações nos exames, e a maioria dos manipuladores $(60,0 \%)$, nunca participou de treinamento quanto às BPM, apenas 4,6\% realizaram no período inferior a seis meses, como determina a RDC/Anvisa n 216/2004 (Tabela 1).

A não realização destes exames representa uma inconformidade com a legislação sanitária RDC/Anvisa $\mathrm{n}^{\circ}$ 216/2004 e trabalhista NR 7/2009 do ministério do trabalho e emprego (BRASIL, 2009). Estas legislações exigem o controle de saúde dos manipuladores de alimentos e deve seguir as diretrizes do Programa de Controle Médico de Saúde Ocupacional - PCMSO, com a realização dos exames de coproparasitólogico semestralmente para aqueles que manipulem diretamente os alimentos ou participem diretamente da distribuição e oferta de refeições.

O Quadro 2 mostra o resultado sobre o conhecimento dos manipuladores quanto as Boas Práticas de manipulação, onde se obteve uma média de $89,16 \%$ de acertos, demonstrando um nível de conhecimento satisfatório sobre a segurança dos alimentos $(>60,0 \%)$.

Quadro 2 - Percentual de respostas corretas dos manipuladores de alimentos dos restaurantes tipo self-service do município de Patos - PB, 2019

\begin{tabular}{|c|c|}
\hline Questões & $\begin{array}{c}\% \text { de } \\
\text { acertos }\end{array}$ \\
\hline $\begin{array}{l}\text { 1. As bactérias podem estar na pele, nariz e } \\
\text { boca de pessoas saudáveis, contaminando } \\
\text { assim os alimentos? }\end{array}$ & 84,6 \\
\hline $\begin{array}{l}\text { 2. Alface e outros vegetais crus podem ter } \\
\text { microrganismos que causam doenças } \\
\text { transmitidas por alimentos? }\end{array}$ & 96,9 \\
\hline $\begin{array}{l}\text { 3. Microrganismos responsáveis por doenças } \\
\text { transmitidas por alimentos crescem à } \\
\text { temperatura ambiente? }\end{array}$ & 86,1 \\
\hline $\begin{array}{l}\text { 4. Alimentos preparados com antecedência } \\
\text { são mais suscetíveis ao crescimento de } \\
\text { microrganismos? }\end{array}$ & 89,2 \\
\hline $\begin{array}{l}\text { 5. O armazenamento de alimentos preparados } \\
\text { próximos a alimentos crus favorece a } \\
\text { contaminação cruzada, que pode causar } \\
\text { DTAs? }\end{array}$ & 89,2 \\
\hline $\begin{array}{l}\text { 6. O armazenamento de alimentos perto de } \\
\text { materiais de limpeza pode favorecer a } \\
\text { contaminação química? }\end{array}$ & 100 \\
\hline $\begin{array}{l}\text { 7. Na recepção dos alimentos é importante } \\
\text { verificar a qualidade? }\end{array}$ & 100 \\
\hline $\begin{array}{l}\text { 8. A presença de contaminação física, como } \\
\text { pregos, cabelos ou parafusos na comida, } \\
\text { representa um risco à saúde? }\end{array}$ & 100 \\
\hline $\begin{array}{l}\text { 9. Podem-se manipular alimentos em caso de } \\
\text { cortes/feridas e queimaduras nas mãos e } \\
\text { braços? }\end{array}$ & 93,8 \\
\hline $\begin{array}{l}\text { 10. Alimentos servidos crus (como saladas) } \\
\text { devem ser sanitizados? }\end{array}$ & 100 \\
\hline $\begin{array}{l}\text { 11. Solução sanitizante para higienização das } \\
\text { hortaliças é preparada com } 1 \text { L de água } \\
\text { potável mais } 1 \text { colher de sopa de água } \\
\text { sanitária? }\end{array}$ & 92,3 \\
\hline
\end{tabular}




\begin{tabular}{|c|c|}
\hline \multicolumn{2}{|c|}{ Continuação... } \\
\hline Questões & $\%$ de acertos \\
\hline $\begin{array}{l}\text { 12. O manuseio inadequado de alimentos } \\
\text { aumenta o risco de contaminação, } \\
\text { porque o manipulador pode transmitir } \\
\text { microrganismos à comida? }\end{array}$ & 96,9 \\
\hline $\begin{array}{l}\text { 13. Alimentos podem ser contaminados por } \\
\text { bactérias quando em contato com outros } \\
\text { alimentos que já foram contaminados? }\end{array}$ & 100 \\
\hline $\begin{array}{l}\text { 14. Superfícies de trabalho, como tábuas e } \\
\text { bancadas, podem ser responsáveis pela } \\
\text { contaminação de alimentos? }\end{array}$ & 98,5 \\
\hline $\begin{array}{l}\text { 15. Uma vez preparados, os alimentos cozidos } \\
\text { devem ser mantidos em temperaturas } \\
\text { abaixo de } 5^{\circ} \mathrm{C} \text { e acima de } 60^{\circ} \mathrm{C} \text { ? }\end{array}$ & 87,7 \\
\hline $\begin{array}{l}\text { 16. Os alimentos devem ser resfriados à } \\
\text { temperatura ambiente antes de serem } \\
\text { armazenados no refrigerador? }\end{array}$ & 15.4 \\
\hline $\begin{array}{l}\text { 17. O congelamento pode reduzir, mas não } \\
\text { pode destruir todas as bactérias que podem } \\
\text { causar doenças transmitidas por alimentos? }\end{array}$ & 83,1 \\
\hline $\begin{array}{l}\text { 18. O processo de higienização ocorre em duas } \\
\text { etapas: limpeza e desinfecção? }\end{array}$ & 96,9 \\
\hline $\begin{array}{l}\text { 19. Depois de manusear carne crua, as mãos } \\
\text { devem ser higienizadas somente com água } \\
\text { e sabão? }\end{array}$ & 64,6 \\
\hline $\begin{array}{l}\text { 20. Sempre lavar as mãos com sabão, água } \\
\text { corrente e sanitizantes depois de usar o } \\
\text { banheiro? }\end{array}$ & 100 \\
\hline $\begin{array}{l}\text { 21. Depois de espirrar, as mãos devem ser } \\
\text { devidamente higienizadas? }\end{array}$ & 100 \\
\hline $\begin{array}{l}\text { 22. Unha lixada com esmalte de cor clara pode } \\
\text { ser usada pelo manipulador de alimentos? }\end{array}$ & 73,8 \\
\hline $\begin{array}{l}\text { 23. Uniformes devem ser lavados uma vez por } \\
\text { semana? }\end{array}$ & 96,9 \\
\hline $\begin{array}{l}\text { 24. Não usar adornos durante o manuseio evita } \\
\text { contaminação? }\end{array}$ & 83,1 \\
\hline $\begin{array}{l}\text { 25. A higienização da caixa d água deve ocorrer } \\
\text { a cada } 6 \text { meses? }\end{array}$ & 100 \\
\hline
\end{tabular}

Fonte: dados da pesquisa.

Em relação a não capacitação dos manipuladores, Devides, Maffei e Catanozi (2014) (88,0\%) e Ferreira e Fogaça (2018) (75,0\%) observaram números superiores, Mello et al. (2010) $(30,1 \%)$ e Souza, Azevedo e Seabra (2018) $(10,0 \%)$ números inferiores, quando comparados aos do presente estudo. Esses números elevados de não capacitação revelam que, independente do tempo e local da pesquisa, existe uma negligência por parte dos proprietários dos estabelecimentos quanto ao aperfeiçoamento de seus colaboradores, em desconformidade com a legislação sanitária vigente.

Ao avaliar o nível de conhecimento dos manipuladores sobre as BPM, observou-se uma dispersão significativa dos resultados perto da média, pois na questão que tratava sobre a correta prática de resfriamento dos alimentos apresentou resultado insatisfatório com 15,4\%. Esta prática pode levar à proliferação de microrganismos que resistiram ao aquecimento, representando um risco a saúde do consumidor.

Outra questão que apesar de ter sido classificado como satisfatório, o conhecimento sobre a higienização adequada das mãos ao manipular carnes cruas, apresentou um baixo percentual $(64,6 \%)$ de acertos. Tendo em vista que a higienização incorreta pode levar a contaminação cruzada dos alimentos.

Souza, Azevedo e Seabra (2018) avaliaram o conhecimento de manipuladores de alimentos restaurantes públicos populares do estado do Rio Grande do Norte e também observaram resultados satisfatórios com média de $72,76 \%$ de acertos.

Apesar do resultado geral ter sido considerado satisfatório, o fator de acerto ao acaso pode também ter influenciado para isso, pois durante a aplicação dos questionários foram observadas situações de manipuladores usando adornos, com lesões/cortes nas mãos, unhas com esmaltes, e responderam corretamente as questões quanto a essas restrições para pessoas que manipulam alimentos. Evidenciando a inexistência ou precariedade de supervisão nesses serviços de alimentação e que nem sempre os conhecimentos teóricos são levados à prática pelos manipuladores de alimentos.

De acordo com Freitas, Calazans e Alchiere (2014), a ausência de qualificação na área é um ponto relevante na conduta inadequada dos manipuladores nas práticas de segurança no manuseio de alimentos. Sabe-se que práticas indevidas na manipulação e conservação de alimentos são fatores agravantes quando se trata de DTA. Por isso é reforçada a importância do treinamento e a educação continuada com os manipuladores envolvidos na produção de refeições, agregados a supervisão diária destes trabalhadores, pois a mudança de práticas exige monitoramento.

\section{Conclusão}

Nas equipes de manipuladores estudadas predominaram mulheres, de grau de instrução mínimo e na faixa etária economicamente ativa, e apresentaram um nível de conhecimento satisfatório, no entanto não o põem em prática. Os estabelecimentos pesquisados não monitoram a saúde dos seus trabalhadores e nem investem em programa de capacitação.

\section{Referências}

BRASIL, Secretaria de Vigilância em Saúde, Doenças Transmitidas por Alimentos (DTA). Portal da Saúde - Ministério da Saúde. 2017. Disponível em: <www.saude.gov.br>. Acesso em: 02 jul. 2019.

BRASIL. Ministério da saúde. Conselho Nacional de Saúde. Resolução $n^{\circ}$ 466, de 12 de dezembro de 2012. Normas regulamentadoras de pesquisas envolvendo seres humanos. Brasília: Conselho Nacional de Saúde, 2012. Disponível em: $<$ http://conselho.saude.gov.br/resolucoes/2012/Reso466>. Acesso em: 20 jun. 2019.

BRASIL, Ministério do Trabalho e Emprego. Norma Regulamentadora. NR-7 - Programa de Controle Médico de Saúde Ocupacional, 2009.

BRASIL. Ministério da Saúde. Agência Nacional de Vigilância Sanitária - Anvisa. Resolução-RDC no. 216, de 15 de setembro de 2004. Dispõe sobre Regulamento Técnico de Boas Práticas para Serviços de Alimentação. Brasília, DF, 2004.

CAMPOS, A.K. et al. Assessment of personal hygiene and practices of food handlers in municipal public schools of Natal, 
Brazil. Food Control, v.20, n.9, p.807-810, 2009. doi: 10.1016/j. foodcont.2008.10.010.

CAVALLI, S.B.; SALAY, E. Segurança do alimento e recursos humanos: estudo exploratório em restaurantes comerciais dos municípios de Campinas, SP e Porto Alegre, RS. Hig. Alim., v.18, n.126/127, p.29-35, 2004.

DEVIDES, G.G.G.; MAFFEI, D.F.; CATANOZI, M.P.L.M. Perfil socioeconômico e profissional de manipuladores de alimentos e o impacto positivo de um curso de capacitação em Boas Práticas de Fabricação. Braz. J. Food Technol., v.17, n.2, p.166-176, 2014. doi: 10.1590/bjft.2014.014.

FERREIRA, Y.G.S.; FOGAÇA, L.C.S. Levantamento do nível de conhecimento dos manipuladores de serviços de alimentação em feiras livres sobre Boas Práticas de Fabricação, na Cidade de Vitória da Conquista - BA. Id on Line Rev. Mult. Psic., v.12, n.40, p.1093-1104, 2018.

FREITAS, J.F.; CALAZANS, D.L.M.S.; ALCHIERE, J.C. Food handlers occupational and professional training characterization. J Nutr Food Sci, v.4, p.1-6, 2014. doi: 10.4172/21559600.1000325 .

MEDEIROS, M.G.G.A; CARVALHO, L.R.F.; MAIA, M.
Percepção sobre a higiene dos manipuladores de alimentos e perfil microbiológico em restaurante universitário. Ciên. Saude Colet., v.22, n.2, p.383-392, 2017. doi: 10.1590/141381232017222.17282015 .

MELLO, A.G. et al. Conhecimento dos manipuladores de alimentos sobre boas práticas nos restaurantes públicos populares do Estado do Rio de Janeiro. Braz. J. Food Technol., v.13, n.1, p.60-68, 2010. doi: 10.4260/BJFT2010130100008.

NIETO-MONTENEGRO, S.; BROWN, J.L.; LABORDE, L.F. Development and assessment of pilot food safety education materials and training strategies of Hispanic workers in the mushroom industry using the Health Action Model. Food Control, v.19, p.616-633, 2008. doi: 10.1016/j.foodcont.2007.07.005.

SOUZA, C.V.S.; AZEVEDO, P.R.M.; SEABRA, L.M.J. Food safety in Brazilian popular public restaurants: Food handlers' knowledge and practices. J Food Saf., v.38, n.5, p.e12512, 2018. doi:10.1111/jfs. 12512 .

VO, T.H. et al. Knowledge, attitudes, practices and training needs of food-handlers in large canteens in Southern Vietnam. Food Control, v.57, p.190-194, 2015. doi: 10.1016/j. foodcont.2015.03.042. 